\title{
Simulation and Characterization of Underwater Target Detection using LIDAR System
}

\author{
\{M. Darwiesh ${ }^{*}$, Ashraf F. El-Sherif, Yasser H. El-Sharkawy, Mahmoud F. Hassan $\}^{\dagger}$
}

\begin{abstract}
LIDAR technology has applications in many different fields like geography, geology, geomantic, archaeology, seismology, atmospheric physics, and optical remote sensing technology. A LIDAR model for submerged target detection is presented which allows the user to identify and localize the presence of underwater targets which have measurable light reflectance.
\end{abstract}

The main idea of the simulation code is to collect the different powers received from many round trip travel times at successive scanning beams which enable the identification of the target in specified working region.

Absorption, scattering, and backscattering of light in different media through which light propagates were taken into consideration to achieve the accurate values of the received power from different target shapes which expresses the real conditions.

Keywords: LIDAR, Underwater, Attenuation, Scattering, Absorption, Chlorophyll.

\section{Introduction}

The simulation for the LIDAR system detector operation is based mainly on a number of theories and equations. The LIDAR range equation is the main law used, which enables the calculation of the received power scattered from an object.

A number of laser beams are used to sweep the area under search, in order to trace the Laser beams as they propagate, Snell's law is used to predict the angles of refraction for each beam at the separating surface between air and sea water which is the sea surface. Fresnel's equation is used to calculate the reflectance and transmittance for each incident beam.

As the signal travels through air and through sea water, the total attenuation of the transmitted and received signals is calculated based on the environmental conditions of both the air and the sea. The received signal power also depends on the properties of the target like shape, dimensions and the reflectivity of its surface.

The main idea of the program is to simulate and collect the different powers received from different round trip times at different scanning rays whose number is determined based on the resolution of scanning of the working area.

\footnotetext{
** M.darweesh@mtc.edu.eg

$\dagger \quad$ Egyptian Armed Forces, Egypt.
} 
The simulation program is divided into three main parts. The first part is responsible for describing the working area. The second part deals with defining the attenuation and backscattering in air and water and any modifications made due to the presence of a target. While the third part deals with calculating the reflected power from the laser beams used to sweep the working area.

The simulation starts at the maximum angle (the near edge of the working area) and will move until arriving to the minimum one (far edge of working area). The simulation is going to collect samples of the power each nanosecond, so the time step between two different samples is a nanosecond. For each scanning ray, it is going to scan until reaching the maximum round trip time ( $\mathrm{rtt}_{\max }$ ), this time is the amount of nanoseconds our beam at the far edge of the working area needs to arrive to the estimated maximum depth in water.

For each ray, the angle of incidence is calculated and based on that angle using both Snell's law and Fresnel's law we can calculate the angle of refraction and the reflectance coefficient and the transmittance coefficient which helps in determining the path of the laser ray after hitting the water surface and also calculate the amount of power reflected back into air and the amount of power transmitted to the water.

For each ray the time needed to reach the water surface is calculated and this helps in determining the location of the power sample whether it is in the air or below water surface thus determines which medium parameters to use (absorption, scattering, backscattering and attenuation).

Once the target is detected, the reflected power is from the outer surface facing the laser source only, then there is no power reaching the inside of the target nor the region beyond the target (target shadow) and the reflected power from that shadow area is equal to zero.

\section{Describing the Working Area}

The initial dimensions of the working area are defined by the simulation conditions, the laser source vertical location $(H)$, the near edge $\left(\mathrm{L}_{\mathrm{n}}\right)$ and the far edge $\left(\mathrm{L}_{\mathrm{f}}\right)$ in addition to the estimated maximum depth reached by laser beams (yemd) in the working area. The resolution (Res) of sweeping the working area determines the number of rays used by the Eqn.1.

$$
\text { Number of samples }=\frac{L_{f}-L_{n}}{\text { Res }}+1
$$

Based on these initial data several extra terms are calculated. At both the near and far edges, the angles of incidence $\left(\theta_{\text {ne }}, \theta_{\text {fe }}\right)$, are calculated by the Eqns. 2-a. and 2-b.

$$
\begin{aligned}
& \theta_{n e}=\tan ^{-1}\left(\frac{H}{L_{n}}\right) \\
& \theta_{f e}=\tan ^{-1}\left(\frac{H}{L_{f}}\right)
\end{aligned}
$$

The distance between the laser source and the edges in air $\left(\mathrm{R}_{\text {air_ne }}, \mathrm{R}_{\text {air_fe }}\right)$ are calculated by Eqns. 3-a. and 3-b.

$$
\begin{aligned}
& R_{\text {air_ne }}=\frac{H}{\sin \left(\theta_{n e}\right)} \\
& R_{\text {air_ne }}=\frac{H}{\sin \left(\theta_{n e}\right)}
\end{aligned}
$$

Angles of refraction at the edges are calculated by using Snell's law Eqns.4-a. and 4-b.

$$
\theta_{\text {snl_ne }}=\sin ^{-1}\left(\frac{n_{a} \sin \left(90-\theta_{n e}\right)}{n_{w}}\right)
$$




$$
\theta_{\text {snl_fe }}=\sin ^{-1}\left(\frac{n_{a} \sin \left(90-\theta_{f e}\right)}{n_{w}}\right)
$$

The round trip time in air the beam takes to reach these edges ( $\mathrm{rtt}_{\text {air_ne }}, \mathrm{rtt}_{\text {air_fe }}$ ) are calculated by the Eqns. 5-a. and 5-b.

$$
\begin{aligned}
& r t t_{\text {air_ne }}=\frac{2 R_{\text {air_ne }}}{c_{a}} \\
& r t t_{\text {air } f e}=\frac{2 R_{\text {air } f e}}{c_{a}}
\end{aligned}
$$

The distance travelled by the beam at the both edges in water is calculated from Eqns. 6-a. and 6-b.

$$
\begin{aligned}
& R_{w_{-} n e}=\frac{y_{\text {emd }}}{\cos \left(\theta_{\text {snl_ne }}\right)} \\
& R_{w_{-} f e}=\frac{y_{\text {emd }}}{\cos \left(\theta_{\text {snl_fe }}\right)}
\end{aligned}
$$

The round trip time at the both edges in water is calculated from Eqns.7-a. and 7-b.

$$
\begin{aligned}
& r t t_{w_{-} n e}=\frac{2 R_{w_{n} n e}}{c_{w}} \\
& r t t_{w_{-} f e}=\frac{2 R_{w_{-} f e}}{c_{w}}
\end{aligned}
$$

The total round trip time in both air and water at far edge is assumed to be the longest round trip time of all beams; it is calculated from Eqn. 8.

$$
r t t_{\max }=r t t_{\text {air } f e}+r t t_{w_{-} f e}
$$

As the laser beam at the far edge propagates in water and therefore it has a horizontal distance component which extends the working area to beyond the far edge. The maximum horizontal distance at the far edge $\left(\mathrm{x}_{\mathrm{mhfe}}\right)$ is calculated using Eqn. 9.

$$
x_{m h f e}=L_{f}+R_{w_{-} f e} \sin \left(\theta_{\text {snl_fe }}\right)
$$

The size of the matrix (where values of attenuation, absorption and scattering for air and water are recorded and saved) is determined using Eqns. 10-a. and 10-b. To obtain accurate estimation of the scattered signals and to better detect the targets, the working area is divided into steps each of 1 decimeter length $(1 \mathrm{dm}=10 \mathrm{~cm})$. The matrix size is 10 times the dimensions under investigation.

$$
\begin{aligned}
& \text { Mat }_{\text {size }_{x}}=\operatorname{round}\left(x_{\text {mhfe }}+\text { error }_{\text {margin }}\right) \times 10 \\
& \text { Mat }_{\text {size }_{y}}=y_{\text {emd }} \times 10
\end{aligned}
$$

The working area is shown in Fig. 1. where all dimensions are clearly illustrated.

\section{Defining the Attenuation in Air and Water}

\subsection{Air Attenuation}

The attenuation in air is dependent on the weather conditions. Eight different states are taken into considerations, where the attenuation coefficient varies from $0.1 \mathrm{~dB} / \mathrm{Km}$ to $9 \mathrm{~dB} / \mathrm{Km}$ [1]. Table 1 summarizes the attenuation of air depending on weather conditions 


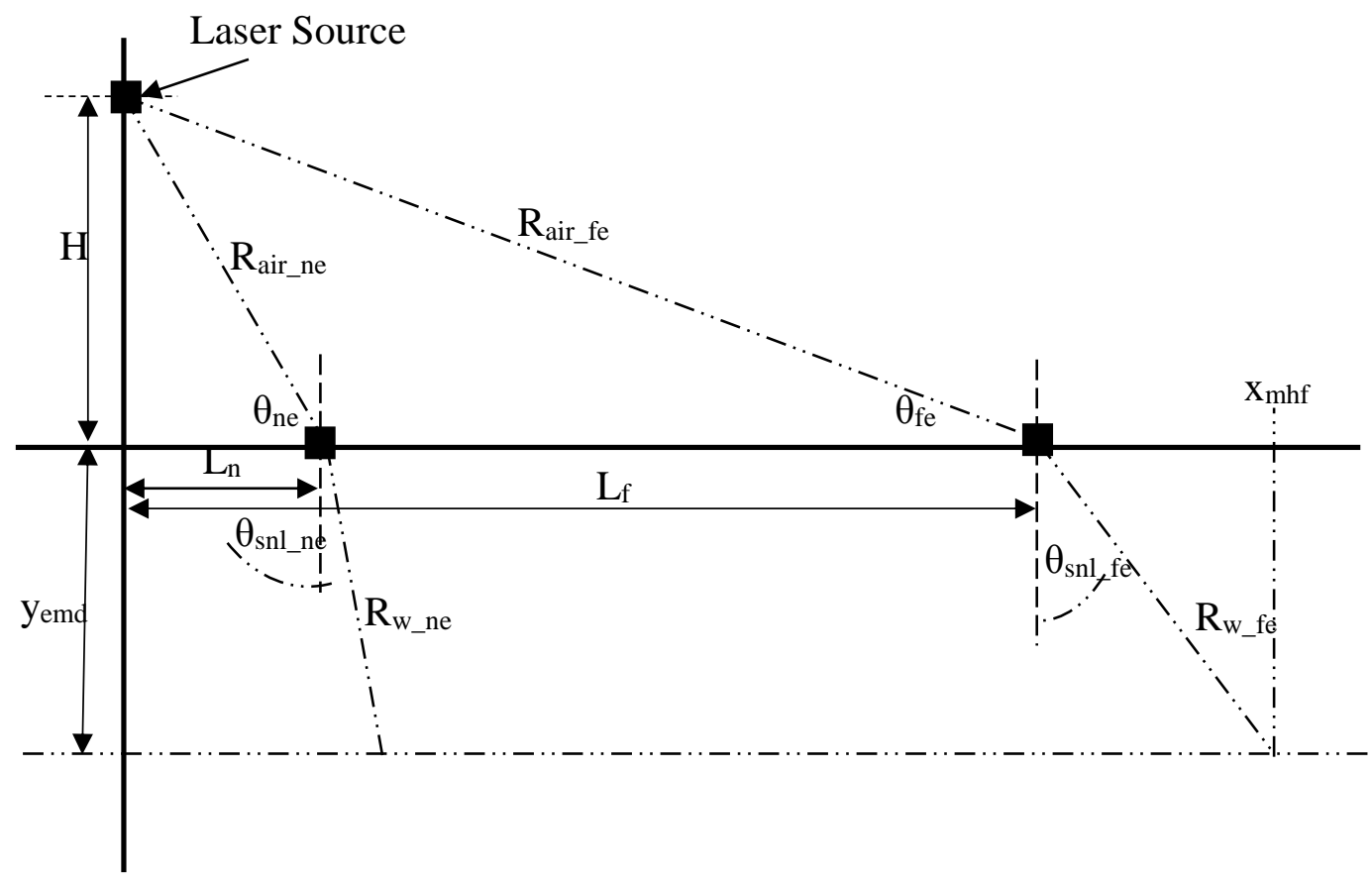

Fig. 1. The dimensions of the working area

Table 1. Air attenuation depending on the weather conditions

\begin{tabular}{|l|c|}
\hline \multicolumn{1}{|c|}{ Weather condition } & Attenuation coefficient (dB/Km) \\
\hline Extremely clear atmosphere & 0.1 \\
\hline Standard Clear atmosphere & 0.5 \\
\hline Clear atmosphere & 1 \\
\hline Light Haze & 1.7 \\
\hline Medium Haze atmosphere & 2.8 \\
\hline Haze atmosphere & 5.2 \\
\hline Medium Rain atmosphere & 5.5 \\
\hline Fog atmosphere & 9 \\
\hline
\end{tabular}

\subsection{Water Attenuation}

The attenuation, transmission loss or the extinction coefficient is estimated with the aid of absorption and scattering coefficients, which are chlorophyll dependent [2].

\subsubsection{Chlorophyll concentration in water}

The main cause of attenuation in water is the presence of organic particles. Chlorophyll pigments absorb light, making coastal waters less clear than open waters. Chlorophyll absorbs light most strongly in the shortest wavelengths (blue and violet) of the visible spectrum. In near- 
shore waters the green wavelength reaches the deepest in the water column. The depthdependent concentration of chlorophyll (measured in $\mathrm{mg} / \mathrm{m}^{3}$ ) is calculated from Eqn. 11 .

$$
C_{(Z)}=C_{o}+\frac{h}{s \sqrt{2 \pi}} e^{-\frac{1}{2}\left(\frac{Z-z_{\max }}{S}\right)^{2}} \quad\left(\mathrm{mg} \cdot \mathrm{m}^{-3}\right)
$$

where $\mathrm{Z}$ is the depth at which chlorophyll concentration is calculated and $\mathrm{C}_{\mathrm{o}}, \mathrm{h}, \mathrm{S}$ and $\mathrm{Z}_{\max }$ are constant parameters

\subsubsection{Absorption coefficient}

Following the bio-optical model of Morel, the absorption coefficient is depth and chlorophyll dependent, as in Eqn. 12.

$$
a_{(z, \lambda)}=\left(a_{w(\lambda)}+0.06 a_{c} C_{(z)}^{0.65}\right)\left(1+0.2 e^{-0.014(\lambda-440)}\right) \quad\left(\mathrm{m}^{-1}\right)
$$

where $a_{w}(\lambda)$ is absorption coefficient of pure water $(=0.052)$.

$\mathrm{a}_{\mathrm{c}}$ is a non-dimensional, statistically derived chlorophyll-specific absorption coefficient $(=0.468)$.

$\mathrm{C}$ is the chlorophyll concentration at the depth of calculation $\mathrm{Z}$.

$\lambda$ is the wavelength used $(532 \mathrm{~nm})$.

\subsubsection{Scattering coefficient}

Morel model to define scattering coefficient is used, Eqn. 13.

$$
b_{(z, \lambda)}=\left(\frac{550}{\lambda}\right) 0.3 C^{0.62}+b_{w(\lambda)} \quad\left(\mathrm{m}^{-1}\right)
$$

where $b_{w}(\lambda)$ is a constant.

$\mathrm{C}$ is the chlorophyll concentration at the depth of calculation $\mathrm{Z}$.

The total attenuation coefficient in water is the sum of both coefficients.

$$
\operatorname{atten}_{(\mathrm{Z})}=\mathrm{a}_{(\mathrm{Z})}+\mathrm{b}_{(\mathrm{Z})} \quad\left(\mathrm{m}^{-1}\right)
$$

\subsection{Backscattering in Water}

Morel distribution is used to calculate its values, Eqn. 14.

$$
B_{(z, \lambda)}=\frac{1}{2} b_{w 1}\left(2 \times 10^{-3}+0.02\left(\frac{1}{2}-\frac{1}{4} \log C\right) \frac{550}{\lambda}\right)\left(0.3 C^{0.62}-b_{w 2}\right)
$$

where $b_{\mathrm{w} 1}$ and $b_{\mathrm{w} 2}$ are constants.

Backscattering in water is dependent on the chlorophyll concentration which in turn is depth dependent.

\section{Calculating the Reflected Power from the Working Area}

The area to be scanned is swept using a number of rays whose number (Eqn. 1.) depends on the resolution of scanning which is the horizontal distance between any two consecutive rays.

For each ray, the whole path is divided into three regions. The first region is through air starting from the laser source and travels till it hits the sea surface. The second region is at the water surface while the third region is through water starting from the point where the ray hit the sea surface till it reaches a depth equivalent to the maximum round trip travel time calculated at the far edge of the working area (Eqn. 8.). 
The ray is then divided into time steps of 1 nanosecond each. For each step of time, the position is calculated and examined to determine whether it is in the air or at sea surface or below sea surface. Then using the appropriate LIDAR equation, the reflected power is calculated.

\subsection{Reflected Power from Air}

The received power from the scattered ray in air is calculated according to the known LIDAR equation [3]

$$
\mathrm{P}_{(\mathrm{air})}=\mathrm{K} \times \mathrm{G}_{(\mathrm{R})} \times \mathrm{B}_{\mathrm{air}(\mathrm{R})} \times \mathrm{T}_{(\mathrm{R})}+\mathrm{n}
$$

where: $\mathrm{K}$ is the system factor.

$\mathrm{G}_{(R)}$ is the geometrical factor.

$\mathrm{B}_{\mathrm{air}(R)}$ is the backscattering in air.

$\mathrm{T}_{(R)}$ is the transmission losses.

$\mathrm{n}$ is the noise associated with the signal.

The system factor $\mathrm{K}$ is equal to

$$
K=\frac{1}{2} P_{o} c_{a} \tau A \eta
$$

where: $\mathrm{P}_{\mathrm{o}}$ is the amount of power within the ray.

$\mathrm{c}_{\mathrm{a}}$ is the speed of light in air.

$\tau$ is the pulse length

A is the effective area of the receiver head

$\eta$ is the system efficiency

The geometrical factor $G_{(R)}$ shown in Fig. 2. includes the laser-beam receiver-field of view overlap function $\mathrm{O}_{(\mathrm{R})}$ which is equal to

$$
O_{(R)}=\left\{\begin{array}{rr}
1 & \text { for } R<1 \mathrm{~km} \\
R & \text { for } R>1 \mathrm{~km}
\end{array}\right.
$$

Therefore

$$
G_{(R)}=\frac{o_{(R)}}{R^{2}}= \begin{cases}\frac{1}{R^{2}} & \text { for } R<1 \mathrm{~km} \\ \frac{1}{R} & \text { for } R>1 \mathrm{~km}\end{cases}
$$

The backscattering $\beta_{\operatorname{air}(R)}$ in air is calculated using the Eqn. 20.

$$
\beta_{\text {air }}=1.39\left(\frac{550}{\lambda}\right)^{4} \times 10^{-8} \quad\left(\mathrm{~cm}^{-1} \mathrm{sr}^{-1}\right)
$$

The transmission losses $\mathrm{T}_{(\mathrm{R})}$ is equal to

$$
T=e^{-2 \alpha R}
$$

where: $\alpha$ is the attenuation of light in air according to the weather conditions discussed in Table 1.

$\mathrm{R}$ is the distance travelled from the laser source to the point at which scattering took place. 


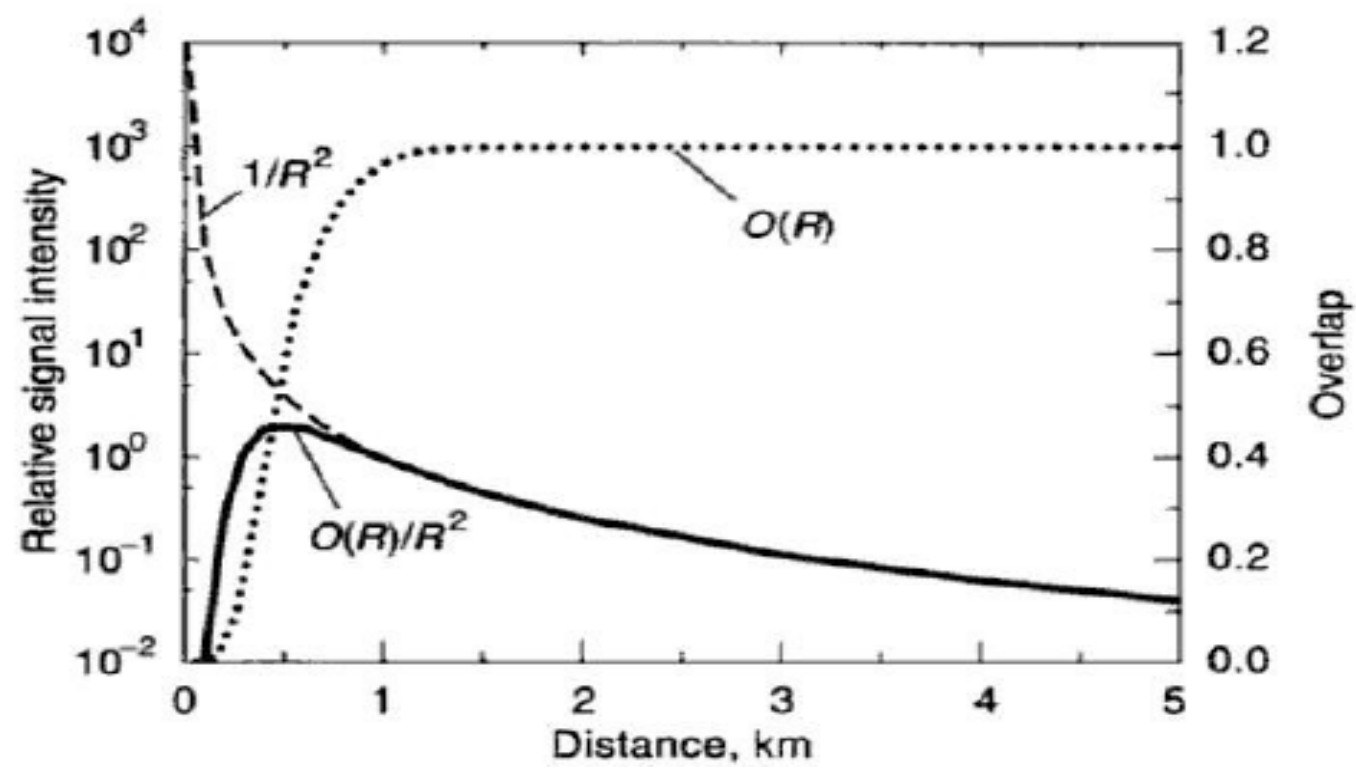

Fig. 2. Geometric factor $\mathrm{G}_{(\mathrm{R})}$

\subsection{Reflected Power from Water Surface}

The discontinuity between air and water splits the laser beam power into two parts, the first part of the signal is scattered back to the air and the other part is transmitted through water. The amount of scattered or transmitted power is calculated with the help of Snell's law and Fresnel's law.

Snell's law calculates the angle of refraction at the point of incidence. The angle is measured between the refracted beam and the normal to the surface, according to the Eqn. 22.

$$
n_{a} \sin \left(\theta_{a}\right)=n_{w} \sin \left(\theta_{w}\right)
$$

where $n_{a}$ is the index of refraction of air.

$\mathrm{n}_{\mathrm{w}}$ is the index of refraction of water.

$\theta_{\mathrm{a}}$ is the angle of incidence.

$\theta_{\mathrm{w}}$ is the angle of refraction.

We use Fresnel's equations to calculate how much power of the incident wave is going to be reflected back to air and how much power is going to be refracted or transmitted to water. The fraction of the incident power that is reflected from the interface is given by reflectance $\Gamma$, and the fraction that is refracted or transmitted is given by transmittance $\mathrm{T}$. The values of $\Gamma$ and $\mathrm{T}$ depends on the polarization of the incident ray.

If the incident light is polarized perpendicular to the incident plane, the reflectance is:

$$
\Gamma_{\text {perpendicular }}=\left[\frac{\sin \left(\theta_{\mathrm{a}}-\theta_{\mathrm{w}}\right)}{\sin \left(\theta_{\mathrm{a}}+\theta_{\mathrm{w}}\right)}\right]^{2}
$$

If the incident light is polarized parallel to the incident plane, the reflectance is:

$$
\Gamma_{\text {parallel }}=\left[\frac{\tan \left(\theta_{\mathrm{w}}-\theta_{\mathrm{a}}\right)}{\sin \left(\theta_{\mathrm{w}}+\theta_{\mathrm{a}}\right)}\right]^{2}
$$

The laser used in the simulation is considered to be polarized parallel to the incident plane.

The received power follows the LIDAR equation, but the reflected power is modified due to the splitting of signal power at the water surface, the reflected quantity is defined by the reflectance term $\Gamma$.

$$
\mathrm{P}_{(\text {water-surface })}=\mathrm{K} \times \mathrm{G}_{(\mathrm{R})} \times \mathrm{B}_{\text {air }(\mathrm{R})} \times \mathrm{T}_{(\mathrm{R})} \times \Gamma+\mathrm{n}
$$




\subsection{Reflected Power from Below Water Surface}

The part of power that penetrates the water and travels through it is calculated from the transmission coefficient

$$
\text { Trans }=1-\Gamma
$$

Backscattering in water constituents (Eqn. 15.) is modified due to the presence of a target, such that the value of backscattering from the target is equal to a constant greater in value than the backscattering of surrounding water constituents, while backscattering from the region behind the target has a value of zero as the target blocks the light from reaching that region.

The location and dimensions of the target are considered by the user of the simulation program. Two types of target cross-section are included in the simulation, the first type is of rectangular cross-section and the second type has a circular cross-section.

Fig. 3. shows the modified scattering coefficient from (a) a target with rectangular cross-section and (b) a target with circular cross-section, both at depth of $10 \mathrm{~m}$.

In Fig. 3. the dark regions are the regions beyond the target where the light rays don't reach, and backscattering from those regions is equal to zero.

The LIDAR equation is used to calculate reflected power from points below water surface as follows

$$
\mathrm{P}_{(\text {water-surface })}=\mathrm{K} \times \mathrm{G}_{(\mathrm{R})} \times \mathrm{B}_{\mathrm{w}(\mathrm{R})} \times \mathrm{T}_{\left(\mathrm{R} \_ \text {air }\right)} \times \mathrm{T}_{\left(\mathrm{R} \_ \text {wat }\right)} \times \text { Trans }+\mathrm{n}
$$

where Trans is the transmission coefficient calculated in Eqn. 26.

$B_{w(R)}$ is equal to backscattering coefficient of water constituents calculated using Eqn.15. and is equal to backscattering coefficient of target if a target exists and is equal to zero for the region blocked by the target.

Since the ray of light travels in both air and water, then the attenuation through air $\mathrm{T}_{\text {air }}$ and attenuation through water $\mathrm{T}_{\mathrm{w}}$ are taken in consideration and included in the LIDAR Eqn. 27.

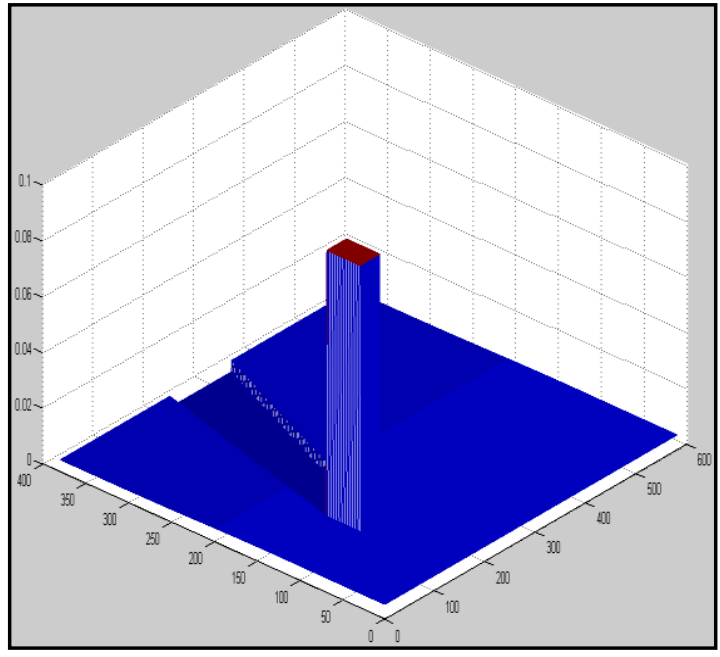

(a)

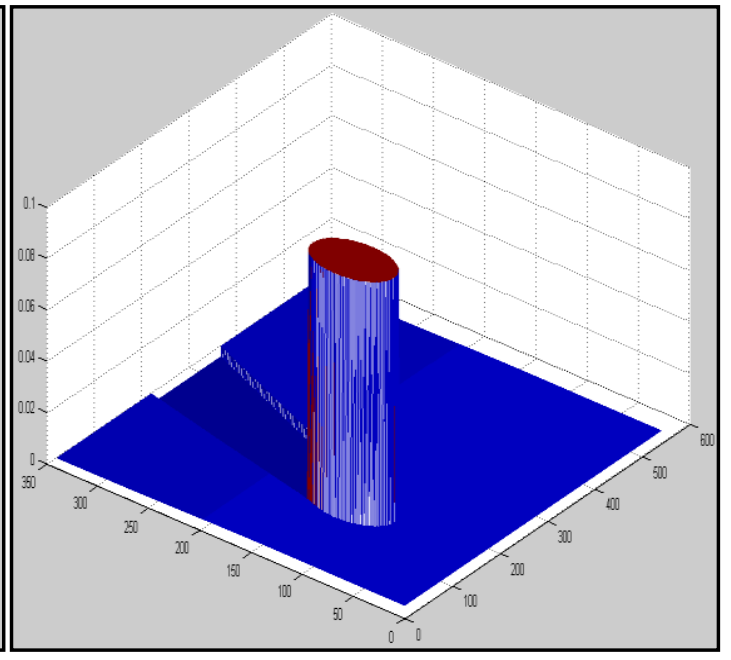

(b)

Fig. 3. Modified backscattering from water due to the presence of

(a) Rectangular cross-section target.

(b) Circular cross-section target. 


\section{Results and Analysis}

The obtained results for the submerged target of circular cross-section with the following input parameters:

The laser source wavelength $(\mathrm{nm}), \lambda=532 \mathrm{~nm}$

The transmitted power (watt), $\mathrm{P}=10^{6} \mathrm{~W}$

The nearest limit of scanned region (meters), $\mathrm{Ln}=10 \mathrm{~m}$

The farthest limit of scanned region (meters), $\mathrm{Lf}=50 \mathrm{~m}$

LIDAR source height (meters), $\mathrm{H}=30 \mathrm{~m}$

The resolution of scanning (meters) $=2 \mathrm{~m}$

Estimated maximum depth in water (meters) $=20 \mathrm{~m}$

We will shoot from 71.565051 degrees $^{\circ}$ to 30.963757 degrees $^{\circ}$.

Number of shots $=21.000000$

The radius of the target (meters), Target Radius $=5 \mathrm{~m}$

The horizontal location of the target center (meters), Target-X-Center $=30 \mathrm{~m}$

The depth of the target center (meters), Target-Y-Center $=10 \mathrm{~m}$

Fig.4. shows the output from the simulation where Fig.4-a represents the scattering distribution vs. depth and Fig.4-b. Represents the absorption distribution vs. depth and Fig.4-c represents the backscattering distribution from all particles in the working area vs. depth and Fig.4-d represents the backscattering distribution in the presence of target vs. depth and Fig.4-e represents the scattering distribution in the presence of target vs. depth and Fig.4-f. represents the total attenuation distribution in the presence of target vs. depth and Fig.4-g. represents the received power distribution from different round trip times at different scanning rays.

What appears in Fig.4-g. as a wall is the reflected power from the water surface. The spikes that appear in the middle are the reflected power from the target surface, which has a much larger value than the surrounding water constituents. A region with zero reflected power appears behind the target, this is the shadow of the target which returns zero power as laser signals are blocked by the target body.
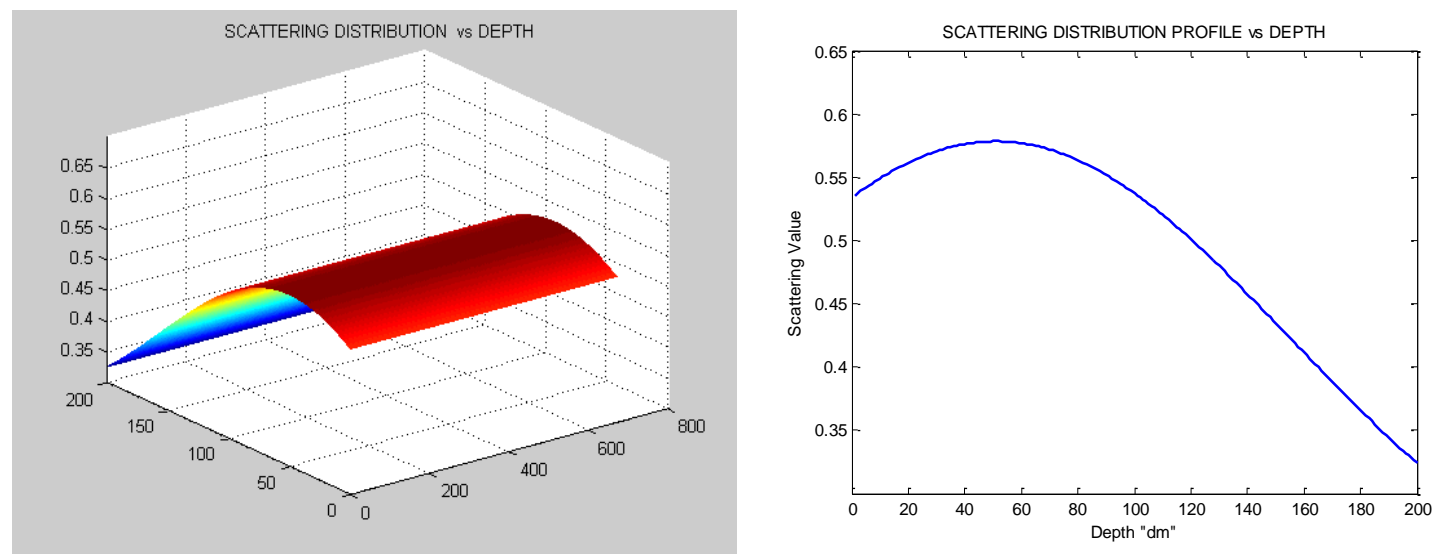

Fig. 4(a). LIDAR simulator output: the scattering distribution vs. depth, 

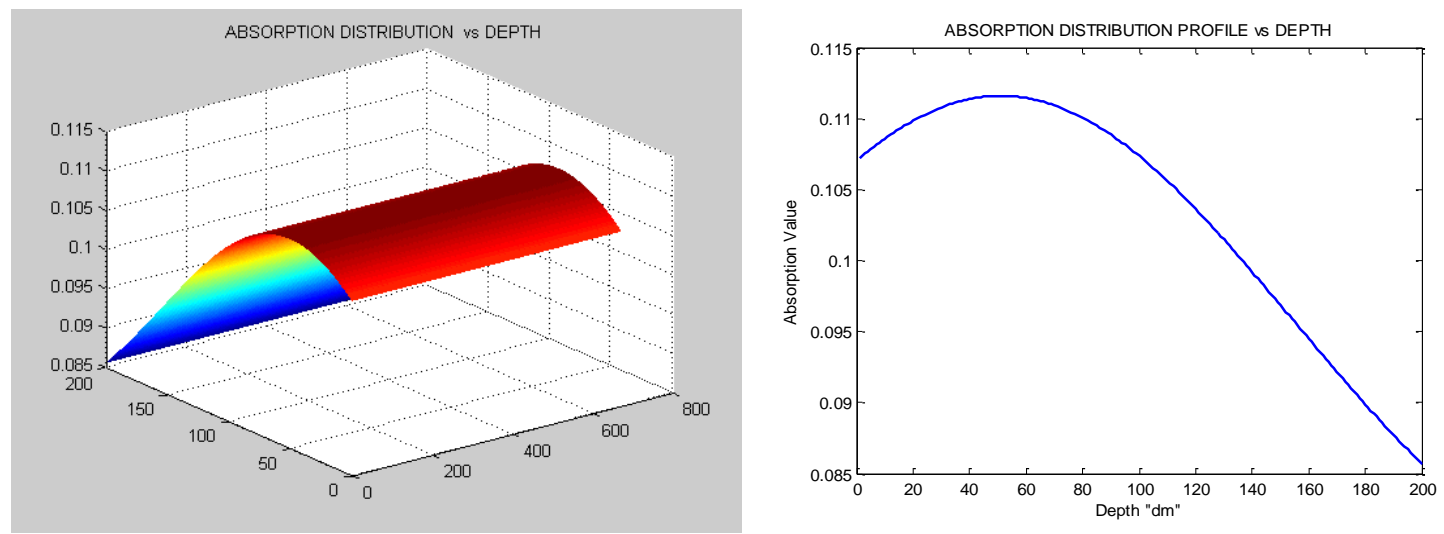

Fig. 4(b). LIDAR simulator output: The absorption distribution vs. depth
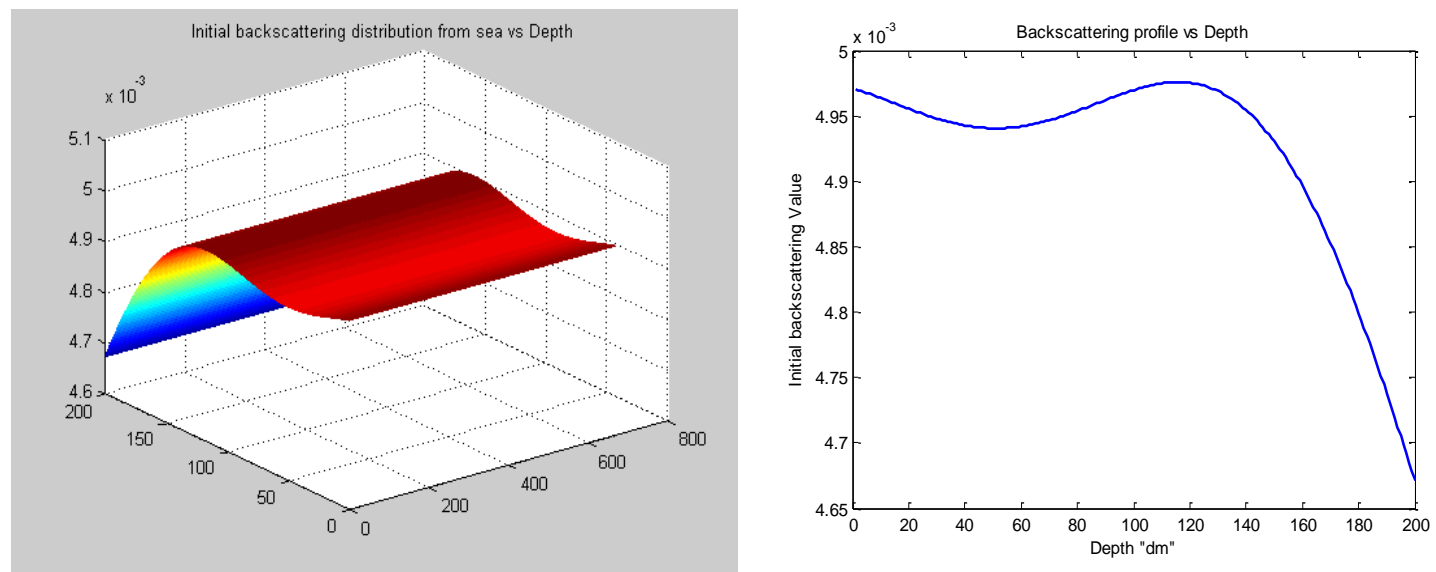

Fig. 4(c). LIDAR simulator output: The backscattering distribution from all particles in the working area vs. depth

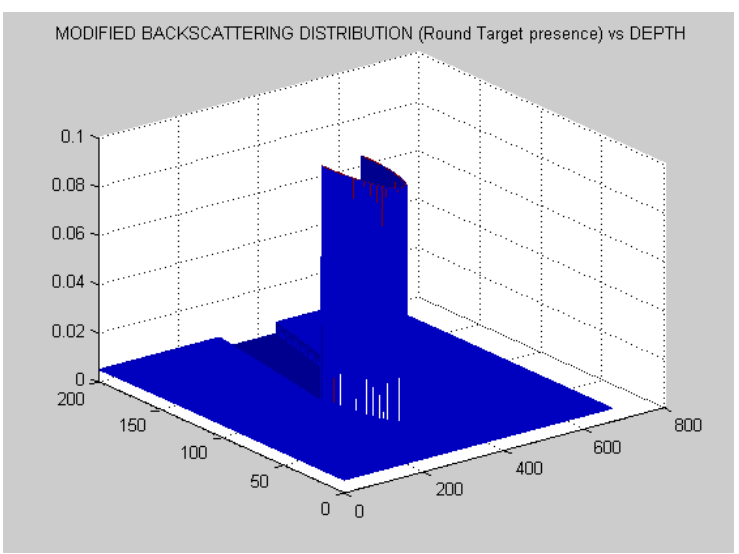

Fig. 4(d). LIDAR simulator output: The Back scattering distribution in the presence of target vs. depth

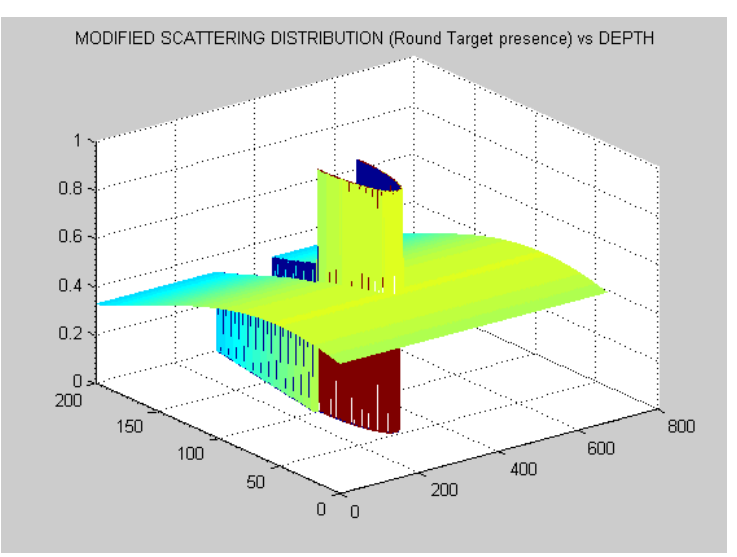

Fig. 4(e). LIDAR simulator output: The scattering distribution in the presence of target vs. depth 


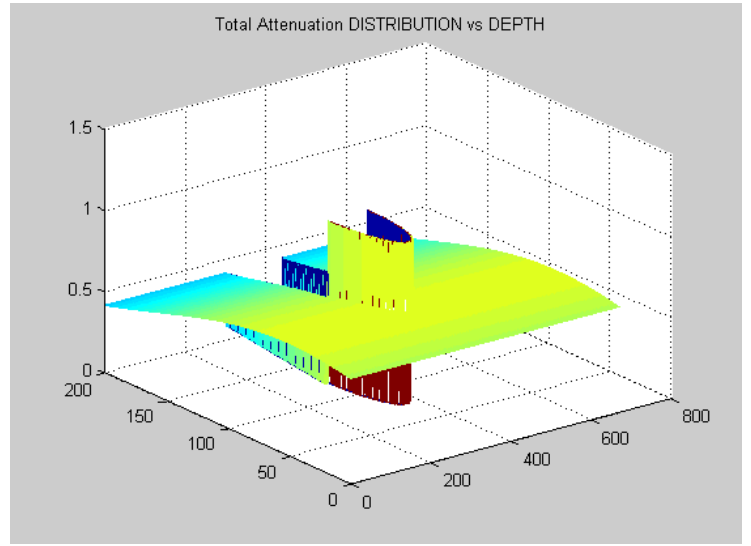

Fig. 4(f). LIDAR simulator output: The total attenuation distribution in the presence of target vs. depth

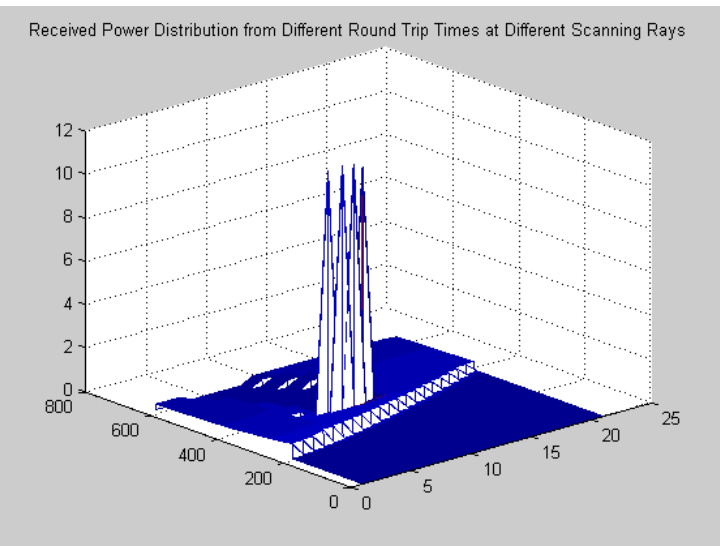

Fig. 4(g). LIDAR simulator output: The received power from different round trip times at different scanning ray

For more details for the same input parameters listed before for the model, Fig. 5. illustrates the reflected power from the first shot in which the sea surface is clearly detected by a sudden increase in the received signal power (0.6watt) after $211 \mathrm{~ns}$ followed by a gradual decrease of the received signal power due to the attenuation of the transmitted signal as it propagates through water and the target not appeared for this shot. If the propagating signal strikes the surface of a target of higher scattering coefficient value than the surrounding water constituents then the amount of scattered power will be higher as wall. This appears in the form of spikes of received power much higher than the neighboring region including the sea surface. The region behind the target will show no received power since the target will block laser signals from reaching beyond the target which is cleared in Fig. 6. Which shows the reflected power from the eighth shot in which the laser beam reaches the water surface after $255 \mathrm{~ns}$ and the beam scattered by target after $315 \mathrm{~ns}$ where the scattered signal from the target is measured to be 12 watt.

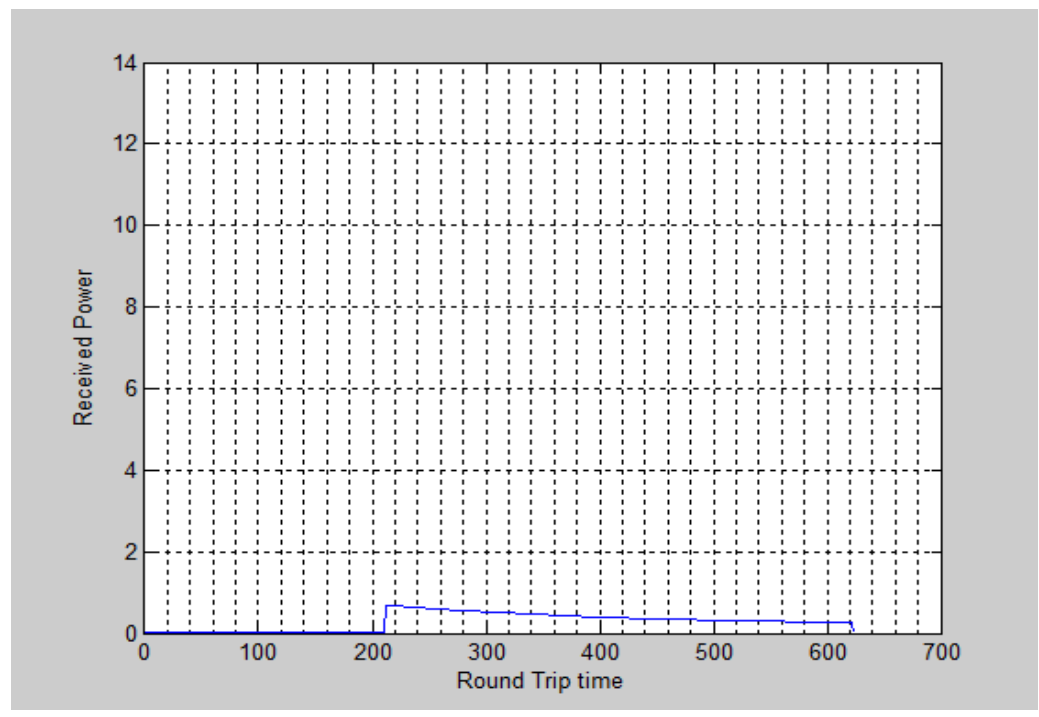

Fig. 5. Received power vs. round trip time for the first shot 


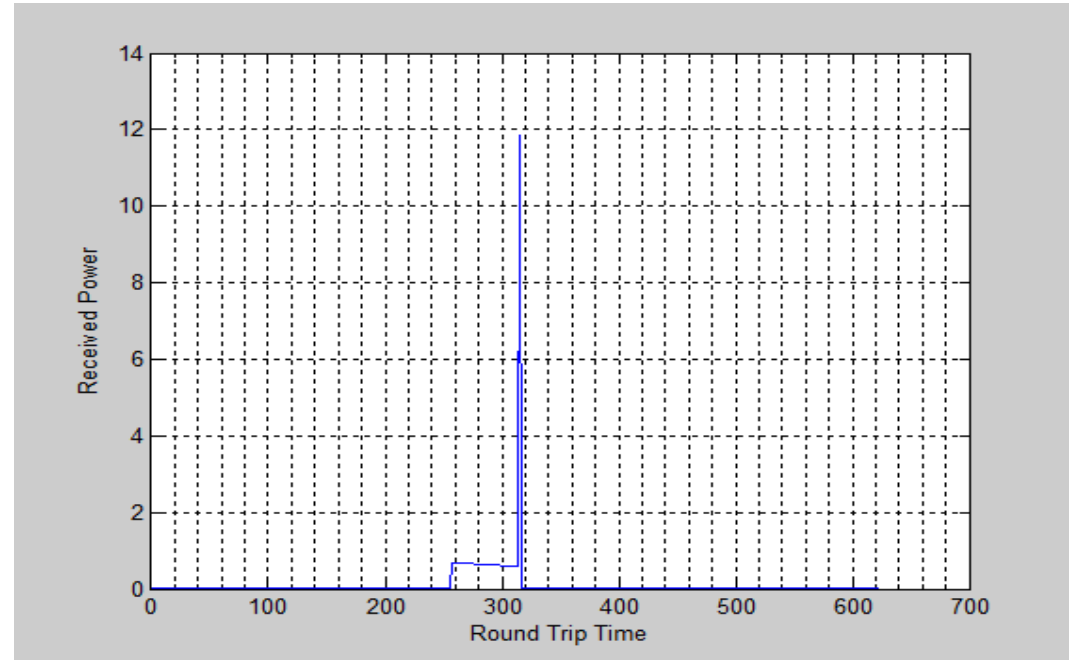

Fig. 6. Received power vs. round trip time for the eighth shot

\section{References}

[1] Jelalian, A.V. (1992), Laser Radar Systems, Artech House, London.

[2] Mobley, C. (1994), Light and Water: Radiative Transfer in Natural Waters, Harcourt Publishers Ltd.

[3] Weitkamp, C. (2005), Lidar, Range-Resolved Remote Sensing of the Atmosphere, Springer. 